\title{
Electroluminescence as internal light source for measurement of the photonic strength of random porous GaP
}

\author{
A. F. van Driel, ${ }^{\text {a) }}$ D. Vanmaekelbergh, and J. J. Kelly \\ Debye Institute, Utrecht University, P.O. Box 80 000, 3508 TA Utrecht, The Netherlands
}

(Received 26 January 2004; accepted 22 March 2004; published online 29 April 2004)

\begin{abstract}
During porous etching of GaP, electroluminescence ranging from the ultraviolet to the near-infrared is generated at the interface of the porous and the nonporous layer. This is used to measure the wavelength-dependent transmission of light through porous layers in a wide thickness range. Two types of porous structures, characterized by different pore sizes, were studied. The transmission of the emitted light gives valuable information about wavelength-dependent diffusion of light through porous GaP. (C) 2004 American Institute of Physics. [DOI: 10.1063/1.1748839]
\end{abstract}

Several $n$-type III-V semiconductors can be made porous by anodic etching. ${ }^{1-7}$ Porous InP, GaAs, and GaP are particularly interesting because they have a considerable band gap and a large refractive index. These materials are therefore important for photonic applications. The structure of the porous layer depends on the etching conditions and the type of semiconductor used. The pores in InP can be either crystallographically oriented or oriented in the direction of the current, depending on the current density. ${ }^{4}$ Porous GaP with a random interconnected structure has been studied extensively. Porous multilayers in GaP, with alternating porosity, can be formed by etching with alternating potential. ${ }^{8}$ In several recent articles diffusive light transport in porous $\mathrm{GaP}$ has been considered. Optimization of the etching process of $\mathrm{GaP}$ has yielded the strongest random-scattering material for visible light reported to date. ${ }^{9}$ The onset of Anderson localization $^{10}$ and anisotropic diffusion ${ }^{11}$ of visible light have been observed.

Recently, it was shown that during etching of GaP hotcarrier luminescence is generated at the interface between the porous and the nonporous material. ${ }^{12}$ A broad spectrum, including a supra-band-gap contribution, was observed. The luminescence was attributed to recombination of electronhole pairs, which were generated by interband tunneling and (subsequent) impact ionization. This internal light source moves with the porous/nonporous interface as the porous layer thickens. Thus, the transmission of the emitted light through porous $\mathrm{GaP}$ can be measured in situ as a function of the layer thickness. In this letter we consider electroluminescence (EL) spectra from which transmission spectra are deduced. The intensity of the emission at various wavelengths is shown as a function of the thickness of the porous layer. The wavelength-dependent scattering properties of the porous layer are described and are related to the structure of the porous layer.

For the experiments $300 \mu \mathrm{m}$ thick $n$-type GaP wafers, supplied by Groupe Arnaud Electronics and Hewlett Packard, with a (100) surface orientation and a (sulphur) doping density of $10-20 \times 10^{17} \mathrm{~cm}^{-3}$ (sample A) and $7 \times 10^{17} \mathrm{~cm}^{-3}$ (sample B) were used. Pieces of $\sim 6 \times 6 \mathrm{~mm}$

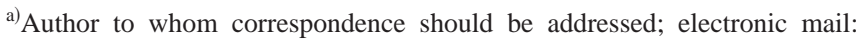
a.f.vandriel@phys.uu.nl
}

were cut and glued on a copper plate with a conductive adhesive paste. A circular area of $0.13 \mathrm{~cm}^{2}$ was exposed to the electrolyte by means of a Teflon sticker. Experiments were performed with the GaP working electrode in a threeelectrode setup, with a platinum counter electrode and a saturated calomel electrode (SCE) as reference. All experiments were performed in the dark, at room temperature in an aque-

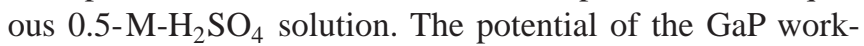
ing electrode was controlled by an EG\&G PAR 273A potentiostat, which was programmed by a computer with homemade software. Luminescence spectra were recorded with a Princeton Instruments CCD camera (liquid-nitrogen cooled, $1024 \times 256$ pixels) in combination with an Acton Pro monochromator (150 lines $/ \mathrm{mm}$, blazed at $500 \mathrm{~nm})$. An Alpha-Step 500 Surface Profiler was used to measure the thickness and the uniformity of the porous layer. The porosity $\phi$ was determined from the charge passed during etching, as described in Ref. 8.

Sample A was etched at $6 \mathrm{~V}$. The porosity was $33 \%$. Sample B was etched at $13.5 \mathrm{~V}$ and had a porosity of $44 \%$. Previous studies showed that etching starts at defect sites at the surface. ${ }^{3,8}$ The current density increased initially and became constant after going through a maximum. ${ }^{8}$ When the current reached its constant level the porous layer had a thickness of $\sim 2 \mu \mathrm{m}$. From then on the thickness of the porous layer increased at a constant rate of 0.47 and $0.62 \mu \mathrm{m} /$ min for samples A and B, respectively. At the end of these experiments the porous layers had a uniform thickness of 80 $\mu \mathrm{m}$. During etching, an EL spectrum was recorded every minute. Figure 1 shows the EL spectra recorded when the porous layer of sample A had a thickness of 4, 10, and 75 $\mu \mathrm{m}$. During etching the applied potential is fixed and the current density remains constant at $8 \mathrm{~mA} / \mathrm{cm}^{2}$. These conditions determine the EL intensity at the porous/nonporous interface. ${ }^{12}$ It is therefore assumed that changes in the EL spectra, as seen in Fig. 1, are due to light scattering and absorption which increase as the porous layer grows in thickness. We will return to this point later. It is clear that emission with an energy larger than the band-gap energy $(\lambda<550$ $\mathrm{nm})$ rapidly vanishes upon increasing thickness of the porous layer. The decrease of the intensity in the sub-band-gap range is much slower and depends strongly on the wavelength of the emission. 


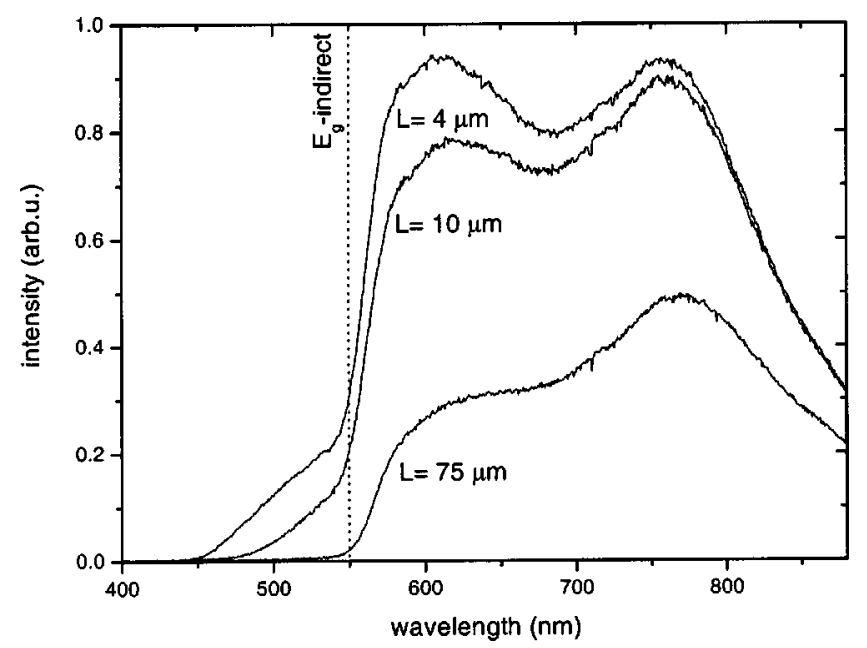

FIG. 1. EL spectra of sample A with a porous layer thickness of 4, 10, and $75 \mu \mathrm{m}$.

It is possible that emitted light reaches the detector after reflection on the backside of the $\mathrm{GaP}$ wafer. The pathlength in the substrate layer, where no scattering occurs, is at least $440 \mu \mathrm{m}$. Emission at a wavelength shorter than $545 \mathrm{~nm}$ is absorbed in the substrate layer while emission at $590 \mathrm{~nm}$ or longer wavelength is not affected by the thickness of the substrate layer. ${ }^{13}$ Obviously, in both cases $(\lambda<545 \mathrm{~nm}$ and $\lambda>590 \mathrm{~nm}$ ) the actual thickness of the substrate layer is not relevant. Thus, in the two regions the only relevant parameter that changes during the etching is the thickness of the porous layer.

A transmission spectrum of the porous layer can be obtained as a function of the thickness by dividing the emitted intensity by a reference intensity. Figure 2 shows transmission spectra for $80 \mu \mathrm{m}$ thick porous layers, together with SEM images $(1 \mu \mathrm{m} \times 1 \mu \mathrm{m})$, for sample A (a) and sample B (b). The pores have a diameter of $51 \pm 11$ and $103 \pm 21 \mathrm{~nm}$ in sample A and sample B, respectively. The two wavelength regions discussed above $(\lambda<545 \mathrm{~nm}$ and $\lambda>590 \mathrm{~nm})$ can clearly be distinguished. At wavelengths shorter than $545 \mathrm{~nm}$ the transmission is very low because of strong absorption in the porous layer. In the sub-band-gap region the transmission increases with wavelength because of the decreasing size pa-

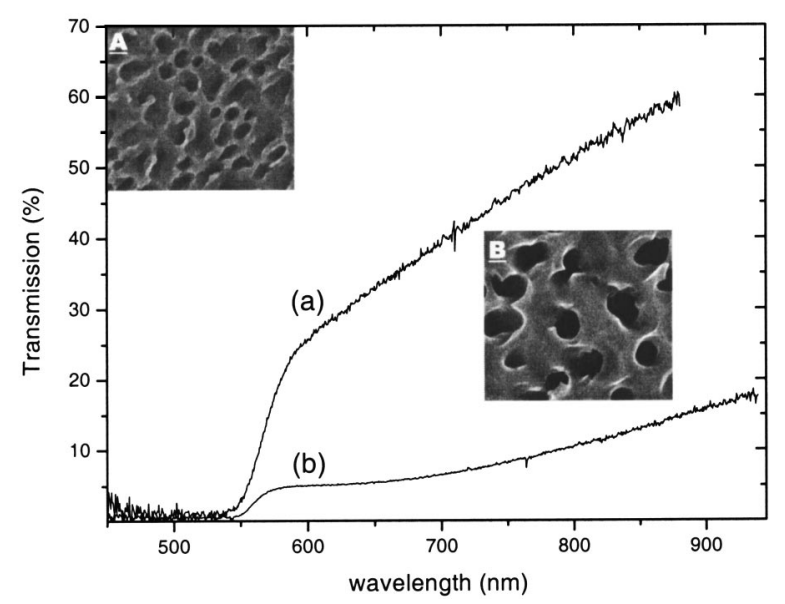

FIG. 2. Transmission spectra of $80 \mu \mathrm{m}$ thick porous GaP layers of (a) sample A and (b) sample B. In the insets SEM pictures $(1 \mu \mathrm{m} \times 1 \mu \mathrm{m})$ of the two samples are shown. ure $3(\mathrm{~b})$ shows the inverse of the emitted EL intensity versus
Downloaded 26 Jul 2004 to 131.211.35.95. Redistribution subject to AlP license or copyright, see http://apl.aip.org/apl/copyright.jsp

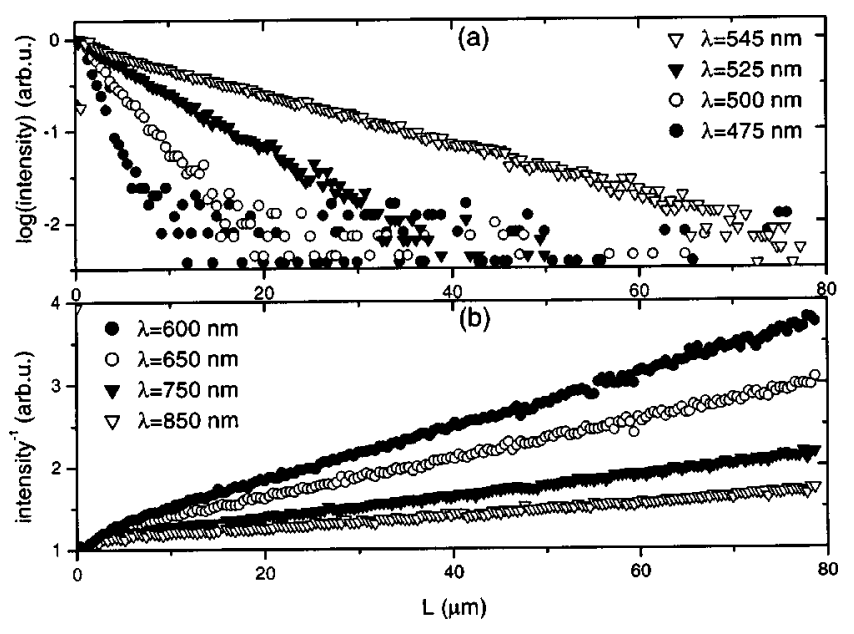

FIG. 3. EL intensity for sample A at various wavelengths with (a) an energy above the band gap and (b) an energy below the band gap as a function of the porous layer thickness: (a) and (b) have the same $x$ axis.

rameter (which is proportional to the ratio of the pore radius to the wavelength). The transmission of sample B is smaller than that of sample A because the size parameter of sample B is larger, i.e., the size of the pores is larger.

For $\lambda<545 \mathrm{~nm}$ both absorption and scattering in the porous layer determine the decrease of the luminescence intensity with thickness. Consequently, an exponential relation between the transmitted intensity and the thickness of the

$$
T_{d}=C \exp \left(\frac{-L}{L_{a}}\right)
$$

with

$$
L_{a}=\sqrt{\frac{l \cdot l_{a}}{3}},
$$

where $T_{d}$ is the diffusive transmission, $L$ the thickness of the porous layer, $L_{a}$ the diffusive absorption length, $l$ is the transport mean free path, $l_{a}$ the absorption length, and $C$ accounts for the reflectivity of the boundaries of the porous layer. Figure 3(a) shows the logarithm of the intensity of the EL versus the thickness of the porous layer. To deduce $l$, the absorption length $\left(l_{a}\right)$ should be known. The absorption length in bulk GaP is well documented. ${ }^{13}$ The absorption length in porous $\mathrm{GaP}$ can be calculated if is assumed that the distance travelled between two scattering events in water is $(l \times \phi)$ and in GaP is $l \times(1-\phi)$. In our case, the typical size of the pores is much smaller than the scattering length. Thus, the absorption length in porous $\mathrm{GaP}$ can be estimated by dividing the absorption length in bulk GaP by $(1-\phi)$. In this way, a transport mean free path of 11 and $4.7 \mu \mathrm{m}$ was found at $545 \mathrm{~nm}$ for samples A and B, respectively.

If the absorption length is much larger than the thickness of the porous layer, the diffusive transmission is given by the following equation: ${ }^{15,16}$

$$
\frac{1}{T_{d}}=\frac{L}{l+C_{1}}+C_{2},
$$

where $l$ is the transport mean free path, and $C_{1}$ and $C_{2}$ contain information on the reflectivity of the boundaries. Figporous layer is expected: ${ }^{14}$ 


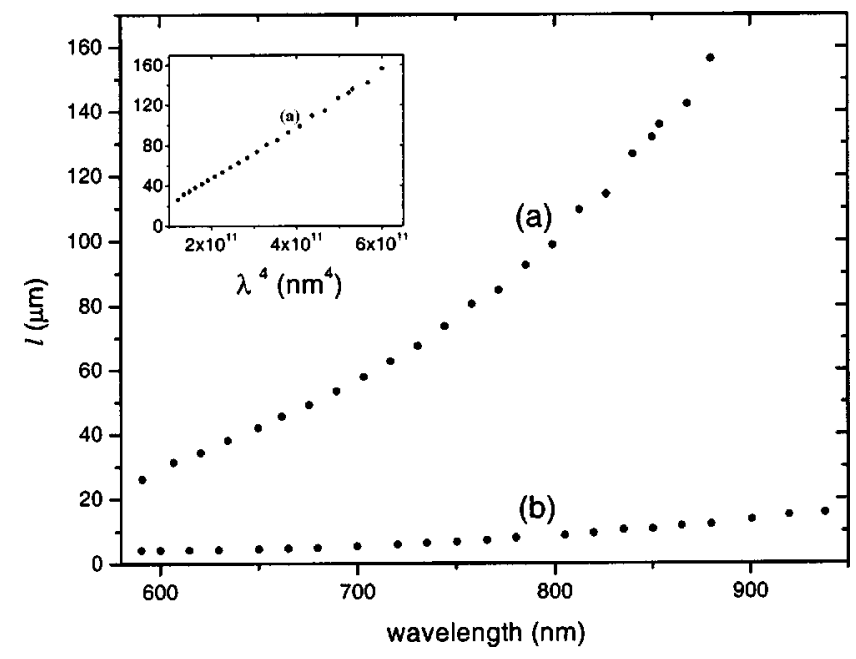

FIG. 4. Transport mean-free-path as a function of wavelength for (a) sample A and (b) sample B. In the inset the transport mean free path is plotted vs $\lambda^{4}$ for sample A.

the thickness of the porous layer. Straight lines are observed, which means that EL is generated at the interface of the porous and the nonporous layer, the porous layer has a uniform structure and that light is scattered in the porous layer without being absorbed. It should be noted that, on occasion, a deviation from straight lines was observed under constant current conditions; this is attributed to an inhomogeneous structure of the porous layer. The inverse of the slopes of Fig. 3(b) is plotted versus the wavelength in Fig. 4.

In the supra-band-gap range scattering is stronger than in the sub-band-gap range. A larger refractive index (3.7 at 475 $\mathrm{nm}$ and 3.2 at $800 \mathrm{~nm}^{13}$ ) and a larger size parameter cause this difference. The difference between samples A and B is ascribed to different pore sizes; larger pores (sample B) scatter light more strongly. Furthermore, it is clear that the size parameter of sample A at wavelengths longer than $590 \mathrm{~nm}$ is considerably smaller 1 . This is the regime of Rayleigh scattering in which the transport mean free path scales with $\lambda,{ }^{4}$ as shown in the inset of Fig. 4. The wavelength dependence observed in Fig. 4 cannot be explained by the wavelength dependence of the refractive index of water, which shows a change of less than $1 \%$ on going from 450 to $900 \mathrm{~nm} .{ }^{17}$ Enhanced backscattering (EBS) measurements on these samples showed that the transport mean free paths are in the micron regime and that sample B scatters light more strongly than sample A. This is in agreement with results obtained here. Formally, the inverse of the slope as plotted in Fig. 4 also contains information on the reflectivity of the boundary of the porous layer. Since the values found with EBS are close to the values found here, it is assumed that $l$ is larger than $C_{1}$ and that the inverse slope is mainly determined by the transport mean free path.

In conclusion we show a nondestructive and simple method to characterize in situ the optical properties of porous $\mathrm{GaP}$. Measuring the intensity as a function of thickness of the porous layer gives valuable information about the structure of the porous layer, the reproducibility of the etching experiment and the scattering properties of the porous layer.

The authors would like to thank B. P. J. Bret for performing EBS measurements and support from the Council of Chemical Sciences (CW) with financial aid from the Netherlands Organization for Scientific Research (NWO).

${ }^{1}$ J. J. Kelly and D. Vanmaekelbergh, The Electrochemistry of Nanomaterials (Weinheim, Germany, 2001), Chap. 4.

${ }^{2}$ H. Foll, S. Langa, J. Carstensen, M. Christophersen, and I. M. Tiginyanu, Adv. Mater. (Weinheim, Ger.) 15, 183 (2003).

${ }^{3}$ B. H. Erne, D. Vanmaekelbergh, and J. J. Kelly, J. Electrochem. Soc. 143, 305 (1996).

${ }^{4}$ S. Langa, I. M. Tiginyanu, J. Carstensen, M. Christophersen, and H. Foll, Electrochem. Solid-State Lett. 3, 514 (2000).

${ }^{5}$ T. Takizawa, S. Arai, and M. Nakahara, Jpn. J. Appl. Phys., Part 2 33, L643 (1994).

${ }^{6}$ P. Schmuki, J. Fraser, C. M. Vitus, M. J. Graham, and H. S. Isaacs, J. Electrochem. Soc. 143, 3316 (1996).

${ }^{7}$ G. Oskam, A. Natarajan, P. C. Searson, and F. M. Ross, Appl. Surf. Sci. 119, 160 (1997).

${ }^{8}$ R. W. Tjerkstra, J. Gómez Rivas, D. Vanmaekelbergh, and J. J. Kelly, Electrochem. Solid-State Lett. 5, G32 (2002).

${ }^{9}$ F. J. P. Schuurmans, D. Vanmaekelbergh, J. van de Lagemaat, and A. Lagendijk, Science 284, 141 (1999).

${ }^{10}$ F. J. P. Schuurmans, M. Megens, D. Vanmaekelbergh, and A. Lagendijk, Phys. Rev. Lett. 83, 2183 (1999).

${ }^{11}$ P. M. Johnson, B. P. J. Bret, J. Gómez Rivas, J. J. Kelly, and A. Lagendijk, Phys. Rev. Lett. 89, 243901 (2002).

${ }^{12}$ A. F. van Driel, B. P. J. Bret, D. Vanmaekelbergh, and J. J. Kelly, Surf. Sci. 529, 197 (2003).

${ }^{13}$ D. E. Aspnes and A. A. Studna, Phys. Rev. B 27, 985 (1983).

${ }^{14}$ J. Gómez Rivas, R. Sprik, C. M. Soukoulis, K. Busch, and A. Lagendijk, Europhys. Lett. 48, 22 (1999).

${ }^{15}$ D. J. Durian, Phys. Rev. E 50, 857 (1994).

${ }^{16}$ J. H. Li, A. A. Lisyansky, T. D. Cheung, D. Livdan, and A. Z. Genack, Europhys. Lett. 22, 675 (1993).

${ }^{17}$ P. Schiebener, J. Straub, J. Sengers, and J. S. Gallagher, J. Phys. Chem. Ref. Data 19, 677 (1990). 